\section{Drosophila Minus is required for cell proliferation and influences Cyclin E turnover}

\author{
Sébastien Szuplewski, ${ }^{1,2}$ Thomas Sandmann, ${ }^{2,4}$ \\ Ville Hietakangas, ${ }^{2,5}$ and Stephen M. Cohen ${ }^{1,2,3,6}$ \\ ${ }^{1}$ European Molecular Biology Laboratory, 69117 Heidelberg, \\ Germany; ${ }^{2}$ Temasek Life Sciences Laboratory, Singapore \\ $117604{ }^{3}$ Department of Biological Sciences, National \\ University of Singapore, Singapore 117604
}

Turnover of cyclins plays a major role in oscillatory cyclin-dependent kinase (Cdk) activity and control of cell cycle progression. Here we present a novel cell cycle regulator, called minus, which influences Cyclin E turnover in Drosophila. minus mutants produce defects in cell proliferation, some of which are attributable to persistence of Cyclin E. Minus protein can interact physically with Cyclin E and the SCF Archipelago/Fbw7/Cdc4 ubiquitin-ligase complex. Minus does not affect dMyc, another known SCF $^{\text {Ago }}$ substrate in Drosophila. We propose that Minus contributes to cell cycle regulation in part by selectively controlling turnover of Cyclin E.

Supplemental material is available at http://www.genesdev.org.

Received May 27, 2009; revised version accepted July 24, 2009.

Progression through the cell cycle requires periodic activation of cyclin-dependent kinases (Cdks) (for review, see Murray 2004). Oscillation in Cdk activity is achieved in part through cyclical synthesis and controlled degradation of cyclins, the regulatory subunits of the Cdks. Cyclin $\mathrm{E}$ is an evolutionarily conserved nuclear cyclin that controls G1/S transition and S-phase progression in animal cells, predominantly by activating Cdk2 (Hwang and Clurman 2005). CycE and $c d k 2$ are essential genes in Drosophila. Cyclin E acts as the limiting factor for G1-Sphase transition (Knoblich et al. 1994; Neufeld et al. 1998; Lane et al. 2000). Cyclin E turnover is important for cell cycle progression and is regulated by a conserved ubiquitin-ligase complex, called SCF (Welcker and Clurman 2008). The SCF complex is built on an elongated scaffold protein, Cullin-1, which recruits the substrate recognition module consisting of Skpl and an F-box protein, as well as a ring domain-containing ubiquitinligase module. Substrate selectivity is mediated by the F-box subunit, in part through recognition of phosphorylated motifs on substrate proteins (Welcker and Clurman

[Keywords: Drosophila; Cyclin E; SCF; cell proliferation; cell cycle] Present addresses: ${ }^{4}$ German Cancer Research Center, Im Neuenheimer Feld, 69120 Heidelberg, Germany; ${ }^{5}$ Institute of Biotechnology, Viikinkaari 9, 00014 Helsinki, Finland

${ }^{6}$ Corresponding author.

E-MAIL cohen@tll.org.sg; FAX 65-6872-7089.

Article is online at http://www.genesdev.org/cgi/doi/10.1101/gad.1822409. Freely available online through the Genes \& Development Open Access option.
2008). The Drosophila F-box protein encoded by archipelago $($ ago $)$ is the ortholog of Fbw7/Cdc4. Scf-Ago promotes degradation of $\mathrm{CycE}$, $\mathrm{dMyc}$, and Trachealess (Moberg et al. 2001, 2004; Mortimer and Moberg 2007).

Here we report the characterization of the classical Drosophila mutant minus. Minus protein can interact physically with Cyclin E and the SCF-Ago complex. Cells lacking Minus fail to degrade $\mathrm{CycE}$, resulting in persistence of CycE. minus mutants show defects in cell proliferation, attributable in part to excess CycE activity, reflecting that normal regulation of $\mathrm{CycE}$ turnover is essential for normal cell proliferation during Drosophila development. We propose that Minus acts as a cell cycle regulator by selectively controlling CycE turnover.

\section{Results and Discussion}

Flies homozygous mutant for a spontaneous mutation in the minus gene $\mathrm{mi}^{1}$ showed small body size, small bristles, and delayed completion of pupal development (Lindsley and Zimm 1992). minus alleles were also isolated in a screen for female sterility (Schüpbach and Wieschaus 1991), and the minus gene has been mapped to the cytogenetic interval 59E on the right arm of the second chromosome (FlyBase Consortium 2003). To isolate new minus alleles, we screened $P$-element insertions in 59E for failure to complement the $m i^{1}$ bristle phenotype. Flies carrying the 1(2)SH0818 P-element insertion in trans to $m i^{1}$ showed a small bristle phenotype, milder than that produced by the combination of $\mathrm{mi}^{1}$ in trans to a deletion (Fig. 1A-C). The stronger mutant combination was also female sterile (Supplemental Table S1). Thus, 1(2)SH0818 appears to have reduced minus activity. 1(2)SH0818 is semilethal, but rare homozygous survivors showed small body size (Fig. 1D) and small bristles. We confirmed that these phenotypes were due to the $P$-element insertion, as flies from which the $P$-element was precisely excised were homozygous viable and normal in size. Animals homozygous for a null allele of minus also showed reduced body size in larval and pupal stages (Fig. 1E,F).

The 1(2)SH0818 P-element is inserted in the $5^{\prime}$ untranslated region (UTR) of the annotated gene CG5360 (Fig. 1G). Two other transposons inserted in this 5' UTR, EY01258 and 1(2)k06908, also produced weak bristle phenotypes in trans to $\mathrm{mi}^{1}$ (Supplemental Table S1), suggesting that they are weak minus alleles. $\mathrm{mi}^{1}$ was isolated as a spontaneous mutation, which can be caused by transposon insertions. We were unable to amplify DNA spanning the second intron of CG5360 from $\mathrm{mi}^{1}$ homozygous animals by PCR, consistent with the possibility that an insertion of DNA disrupts the CG5360 transcription unit (other parts of the gene amplified normally). We generated an additional mi allele by imprecise excision of the viable $P$-element insertion $E Y 01258 . \mathrm{mi}^{\Delta E Y 22}$ is a deletion that removes the two first exons and part of the third exon of CG5360 (Fig. 1G). $m i^{\Delta E Y 22}$ produced phenotypes equivalent to those of a larger deletion that completely removes the gene (Supplemental Table S1), and so behaves genetically like a null allele. Homozygous $m i^{\Delta E Y 22}$ mutants died mainly during early larval stages. The remaining mutants showed a developmental delay and reduced growth rate. After $5 \mathrm{~d}$, the largest mutant larvae were much smaller 

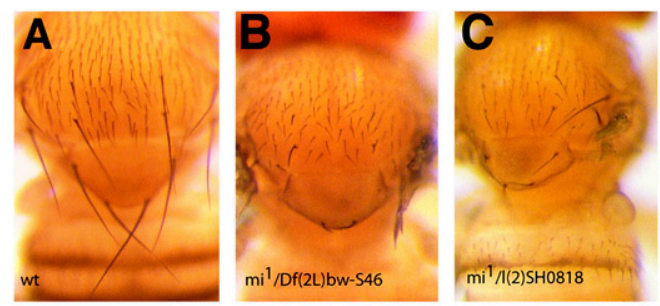

G
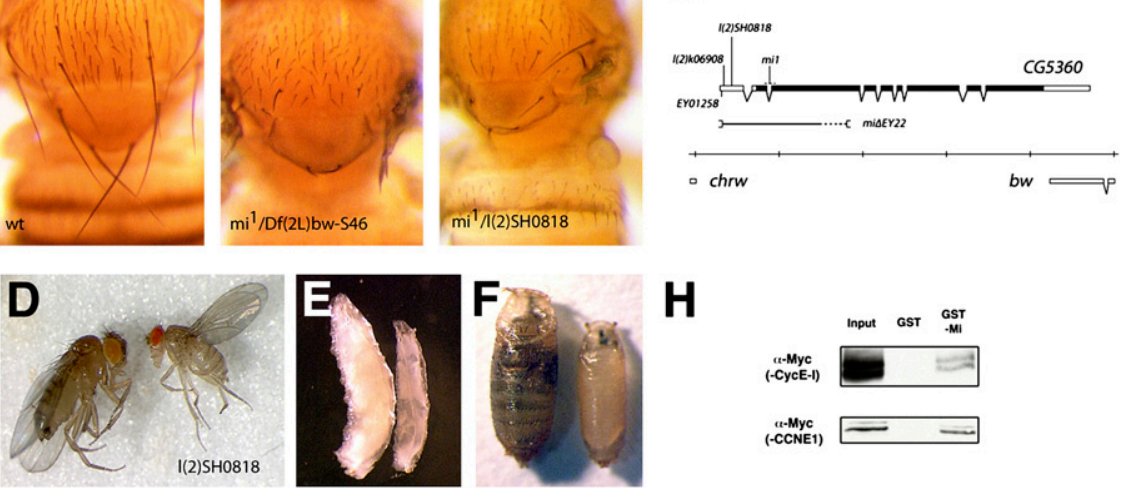

H

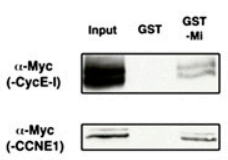

Figure 1. Genetic and molecular characterization of the minus gene. $(A-C)$ Adult dorsal thorax preparations of wild-type (wt) control $(A), \mathrm{mi}^{1} / \mathrm{Df}(2 \mathrm{~L}) \mathrm{bw}$-S46 (B), and $\mathrm{mi}^{1} / 1(2) S H 081$ (C flies. (D) $1(2)$ SH0818/+ control (left) and homozygous $1(2) S H 0818$ mutant females (right). $(E, F)$ Homozygous $y^{1} w^{1118}$ control (left) and $m i^{\Delta E Y 22}$ mutant larvae (right) after 5 and $11 \mathrm{~d}$ of growth in uncrowded conditions. $(G)$ The minus locus showing transgene and transposon insertion sites and the deletion generated by imprecise excision. The ORF is shaded. $(H)$ Lysates from S2 cells expressing Myc-tagged CycE-I or Myc-tagged CCNE1 were assayed for binding to GST-Minus beads or control GST beads. Proteins bound to the beads were detected on immunoblots probed with anti-Myc tag antibody. The input lane represents $8 \%$ (top) and $5 \%$ (bottom) of the total lysate.

than comparably aged control larvae (Fig. 1E). By $11 \mathrm{~d}$, the surviving mutants that had pupated were also small (Fig. 1F).

Minus protein lacks domains of known function, but was identified as a CycE-interacting protein in a genomewide yeast two-hybrid screen (Giot et al. 2003). Ten cyclin-binding sites are predicted using the Eukaryotic Linear Motif server (http://elm.eu.org; Supplemental Fig. S1). Some of the predicted cyclin-binding sites are conserved in other insects-Anopheles gambiae, Tribolium castaneum, and Apis mellifera-but none resides in a region of sequence conservation sufficient to permit identification of an orthologous protein outside of the insects. We confirmed the interaction between Minus and CycE in vitro using GST pull-down assays. The Drosophila Cyclin E gene encodes two proteins that differ in their $\mathrm{N}$ termini (Richardson et al. 1993). A GST-Minus fusion protein was able to bind CycE-I from lysates of S2 cells transfected to express Myc-tagged CycE-I (Fig. 1H). No binding was seen to the GST control. Similar results were obtained with Myc-tagged Drosophila CycE-II protein (data not shown) and with Myc-tagged human CycE isoform 1 (CCNE1) (Fig. 1H).

Interaction with $\mathrm{CycE}$ and its mutant phenotypes suggested that Minus might affect the cell cycle. To test this, we asked if Minus is required for mitotic cell cycle progression by FACS analysis on clones of minus mutant cells and by antibody staining for cell cycle markers in the wing imaginal disc. Under normal circumstances, clones of cells lacking Minus are eliminated by competition with faster-growing Minus-expressing cells (Fig. 5, below). To circumvent this problem, we provided homozygous $m i^{\Delta E Y 22}$ cells with a relative growth advantage by inducing the clones in a Minute mutant background to reduce cell competition (Morata and Ripoll 1975). Homozygous $\mathrm{mi}^{\Delta E Y 22}$ mutant clones were recovered under these conditions. Labeling with an antibody to the G2/Mphase phosphorylated form of Histone $\mathrm{H} 3$ (Su et al.
Homozygous minus mutant imaginal discs were small with elevated levels of cell death, visualized by an antibody to the activated form of Caspase 3 (Fig. 2D,E). To test if cell death contributes to the cell cycle defects in minus mutant clones, we made use of the Drosophila Inhibitor of Apoptosis Protein-1 (DIAP-1). A UAS-DIAP-1 transgene was expressed under control of engrailed-GAL4 with either a UAS-GFP transgene or a UAS-RNAi construct to target Minus for depletion. Expression of the minus RNAi transgene increased the number of cells labeling with anti-PH3 (Fig. 2F), beyond the modest increase observed in the cells expressing DIAP-1 and GFP (Fig. 2G). Thus, accumulation of cells in $G / 2 M$ does not appear to reflect compensation for the elevated level of cell death in the minus mutant.

In Drosophila, most larval tissues grow using an unusual cell cycle called endoreplication, which consists of $S$ and $G$ phases but omits $M$ phase (Lilly and Duronio 2005). In this way, larval cells increase their ploidy and size. To test if the growth defects reflect a defect in endoreplication, homozygous $m i^{\Delta E Y 22}$ mutant salivary gland cells were made using FLP/FRT-mediated recombination. Mutant cells were small, with small nuclei (Fig. $2 \mathrm{H}$, arrows). There was no change in the appearance of minus mutant cells that expressed the baculovirus inhibitor of apoptosis, p35 (Hay et al. 1994), and GFP (Fig. 2I). Thus, the small nuclear size is unlikely to reflect cells undergoing apoptosis, and more likely reflects underreplication of DNA in the polyploid salivary gland cells. These results suggest that minus is required cell-autonomously to promote endoreplication. In this context, it is interesting that preventing CycE oscillation has been shown to impair endoreplication (Follette et al. 1998; Weiss et al. 1998).

Does Minus act by influencing the level of Cyclin E expression? Depleting minus by RNAi in salivary glands abolished the normal pattern of CycE oscillation and resulted in a uniform elevated level of anti-CycE labeling (Fig. 3A,B). Minus RNAi produced a milder version of the endoreplicative defect seen in mutant clones (cf. Figs. 3A 

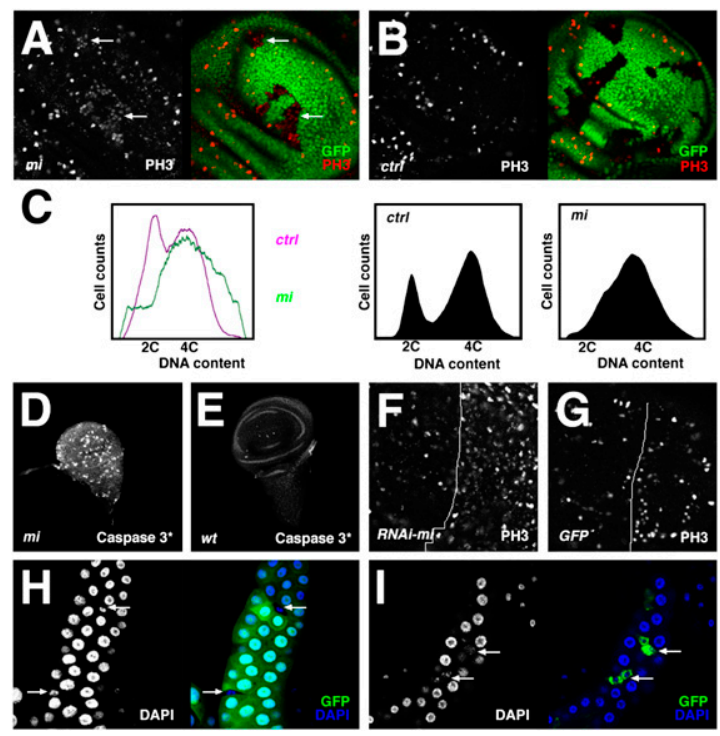

Figure 2. minus is required for cell proliferation and endoreplication. $(A, B)$ Anti-PH3 of third instar wing discs containing clones of $\mathrm{mi}^{\Delta E Y 22}$ mutant cells $(A)$ or wild-type cells $(B)$, each in a Minute background. Clones are labeled by the absence of GFP (arrows). (C) FACS analysis of cells from dissociated Minute/+ heterozygous wing discs containing $m i^{\Delta E Y 22}$ homozygous mutant clones. (Left) Profile of homozygous $\mathrm{mi}^{\Delta E Y 22}$ mutant cells and heterozygous Minute $/ \mathrm{mi}^{\mathrm{I}^{L} \mathrm{EY} 2}$ "control" cells from the same discs. FACS analysis of cells from $y^{1} w^{1118}$ (crtI) and homozygous $\mathrm{mi}^{\mathrm{AEY} 22}$ mutant discs $(\mathrm{mi})$. $(D, E)$ Homozygous $m i^{\Delta E Y 22}$ mutant and control wing imaginal discs labeled with antibody to activated Caspase 3, to visualize apoptotic cells. $(F, G)$ Anti-PH3 staining (PH3) of third instar wing discs expressing UAS-DIAP1 and UAS-RNAi-mi $(F)$ or UAS-GFP $(G)$ in the P compartment (right of the white line). $(H, I)$ Salivary glands containing homozygous $m i^{\Delta E Y 22}$ mutant cells. Mutant cells are marked by the absence of GFP (arrows) DAPI-labeled nuclei are shown in the left panel. (I) $\mathrm{mi}^{4 E Y 22}$ mutant cells expressing UAS-p35 are marked by GFP (arrows).

and $2 \mathrm{H}$ ). To test whether Minus also regulates CycE in mitotic cells, we produced clones of cells mutant for the null allele $m i^{\Delta E Y 22}$. Homozygous $m i^{\Delta E Y 22}$ mutant clones showed elevated levels of CycE protein (Fig. 3C). This was not seen in control clones produced in a Minute mutant background (Fig. 3D). Depleting minus by RNAi was also effective in S2 cells (Fig. 3E) and led to increased CycE levels (Fig. 3F). Comparable results were obtained with an independent dsRNA sequence to deplete minus and by depletion of Archipelago, a core component of the SCF complex that promotes CycE turnover (Fig. 3F). These observations indicate that $\mathrm{CycE}$ is overexpressed in cells with reduced Minus activity.

CycE levels are subject to positive autoregulatory feedback control. Elevated CycE activity, through phosphorylation of $\mathrm{Rb}$, leads to activation of E2F, which in turn transcribes CycE (Du et al. 1996). Quantitative RTPCR showed that depletion of minus led to increased CycE mRNA levels in S2 cells (Supplemental Fig. S2). To bypass this transcriptional feedback, we tested the effect of depleting Minus on a Myc-tagged CycE-I transgene expressed using a heterologous promoter. Depleting Minus in S2 cells increased the level of Myc-tagged CycE-I or Myc-tagged human CCNE1 (Fig. 3G) and Myc-tagged CycE-II (data not shown). We also observed elevated Myc-tagged CycE-I levels in $\mathrm{mi}^{\Delta E Y 22}$ mutant clones produced in a transgenic fly expressing Myc-CycE under control of the hsp70 promoter, which should not be
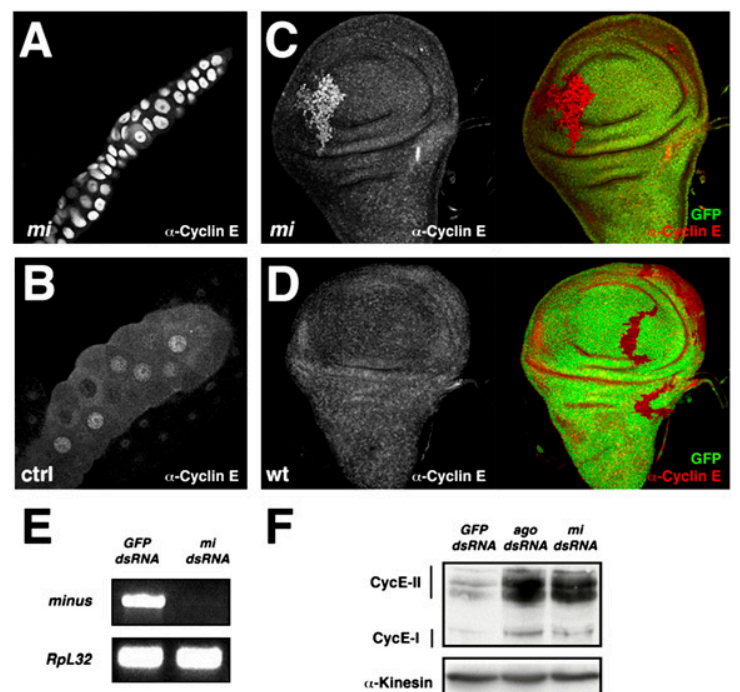

F
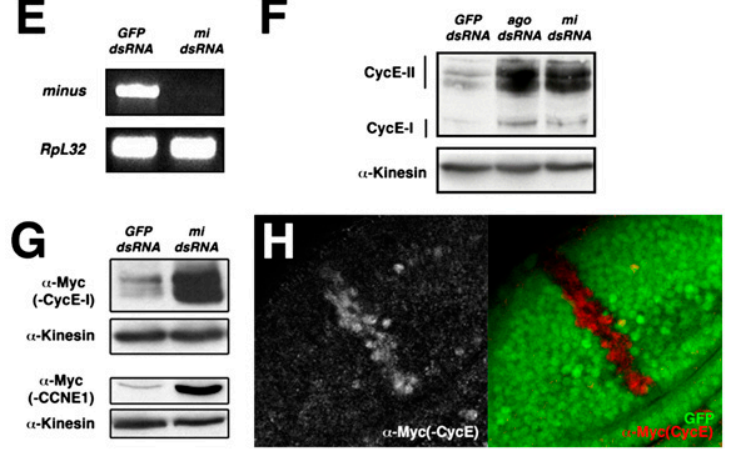

I

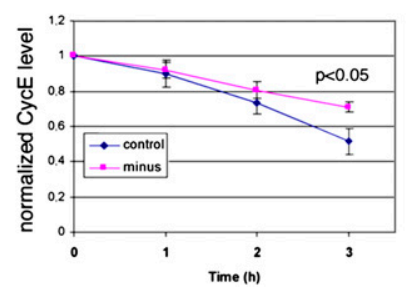

Figure 3. minus mutant affects CycE turnover. $(A, B)$ Anti-CycE staining of third instar salivary glands expressing either UAS-RNAi minus (mi) $(A)$ or UAS-GFP (ctrl) $(B)$ under ptc-GAL4 control. $(C, D)$ Anti-CycE staining of third instar wing discs containing clones of homozygous $m i^{\Delta E Y 22}$ mutant cells or wild-type cells, both in a Minute background to give the clone a relative growth advantage. Clones are labeled by the absence of GFP. (E) RT-PCR to assess the efficiency of minus depletion in S2 cells treated with dsRNA directed to minus or to GFP as a control. The PCR primers were from a region of minus mRNA that did not overlap the region used for the dsRNA. RpL32 was amplified as a control. $(F)$ S2 cells treated with dsRNA to deplete GFP, archipelago, or minus, analyzed by immunoblotting with anti-CycE. (Top panel) Probed with anti-Myc antibody to detect myc-tagged CycE. (Bottom panel) Blot reprobed with anti-Kinesin as a loading control. $(G)$ Extracts from S2 cells transfected to express Myc-tagged-CycE-I or Myc-tagged-Human CCNE1 treated with dsRNA to GFP or minus and analyzed by immunoblotting with anti-Myc to detect the tagged proteins. Blots reprobed with anti-Kinesin as a loading control. $(H)$ Anti-Myc tag staining of a third instar wing disc heterozygous for hsp70-MycCycE-I and a Minute mutation, containing a large clone of $m i^{\Delta E Y 22}$ mutant cells, labeled by the absence of GFP. Note the elevated level of Myc-tagged CycE in the clone. (I) S2 cells expressing CycE-Renilla luciferase were treated with $10 \mu \mathrm{g} / \mathrm{mL}$ cycloheximide, and samples were collected at the indicated times. Cells had been treated $5 \mathrm{~d}$ previously with dsRNA to minus or GFP as a control. Error bars indicate standard deviation of three independent experiments. By $3 \mathrm{~h}$ of cycloheximide treatment, the difference in CycE levels was significant $(P<0.05)$ by Student's $t$-test. Note that use of an unsynchronized cell population might cause us to underestimate the magnitude of the effect. 
subject to the same transcriptional regulation as $C y c E$ (Fig. $3 \mathrm{H}$ ). Together, these results suggest that the direct effects of Minus on CycE levels are post-transcriptional.

To test if the increased level of CycE reflects reduced turnover, we compared the stability of CycE in control cells and in cells depleted of Minus by RNAi. To permit an accurate quantitative assessment of CycE levels, we used a CycE-Renilla luciferase fusion protein expressed in S2 cells (Fig. 3I). In control cells, the level of CycEluciferase dropped by $\sim 50 \%$ during $3 \mathrm{~h}$ of cycloheximide treatment to block new protein synthesis. In Minusdepleted cells, the level of CycE-luciferase decreased by $\sim 30 \%$. The data represent the average of three independent experiments $(P<0.05$ at the 3 -h time point $)$. There was no effect of depleting Minus on Renilla luciferase protein alone (data not shown). Thus, the rate of CycE protein turnover is reduced in cells with reduced Minus activity.

The relationship between $\mathrm{CycE}$ and Minus is reminiscent of that between $\mathrm{CycE}$ and Archipelago, which promotes CycE turnover. So, we asked whether Archipelago and other components of the SCF complex physically interact with Minus. GST-Minus was able to pull down V5-tagged Archipelago, HA-tagged Skp1, and Myc-tagged Cullin1 from lysates of S2 cells (Fig. 4A). None of the proteins associated with the GST control.

We next asked if CycE, Minus, and Ago-SCF form a ternary complex. To allow quantitative measurement of CycE levels, we used the Cyclin E-luciferase fusion protein. Renilla luciferase was used as a control. In addition, the cells were cotransfected with HA-tagged Archipelago and either a GFP-Minus fusion protein or a GFP control. Cell lysates were immunoprecipitated with anti-HA to pull down the Archipelago-SCF complex and associated proteins. The precipitated complex was released from the antibody by incubation with HA peptide, and the eluate containing the resolubilized complexes was subjected to a second round of immunoprecipitation with anti-GFP to pull down GFP-Minus, or GFP in the control. After the sequential immunoprecipitation, Renilla luciferase was measured (Fig. 4B). Control cells transfected to express GFP together with the CycE-luciferase fusion protein showed a very low level of recovered luciferase activity. Luciferase activity was $>40$-fold higher in cells transfected to express GFP-Minus with CycE-luciferase. The data represent the average of four independent experiments, and the observed difference was statistically significant $(P<0.05$ using either Student's $t$-test or Wilcoxon rank sum test). Because the complex was first pulled down with an antibody to tagged Archipelago, followed by an antibody to tagged Minus, recovery of CycE-luciferase depends on the existence of a complex containing all three proteins.

In addition to promoting CycE turnover, the Archipelago-SCF complex promotes turnover of dMyc and Trachealess proteins. dMyc levels were elevated in ago mutant clones (Fig. 4C; Moberg et al. 2004). As dMyc is required for normal cell growth (Johnston et al. 1999), we asked if Minus might also promote dMyc turnover. Clones of $m i^{\Delta E Y 22}$ mutant cells did not show elevated dMyc (Fig. 4D). This suggests that the Minus-containing Ago-SCF complex might be specific for CycE degradation.

We next asked to what extent elevated CycE levels contribute to the phenotypes observed in minus mutants. Bristle progenitors grow by endoreplication, and elevated CycE expression can reduce bristle growth (Weng et al.
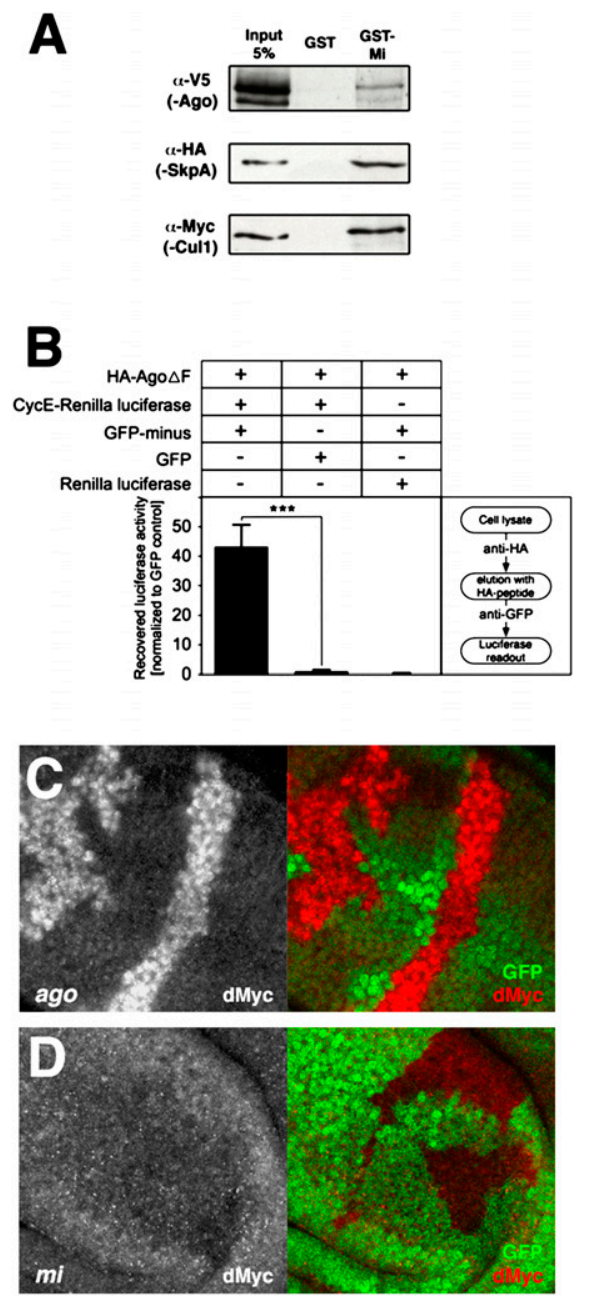

Figure 4. Minus can interact with the SCF-Archipelago complex. (A) Extracts from S2 cells expressing V5-tagged Archipelago, HAtagged SkpA, or Myc-tagged Cull were assayed for binding to GSTMi beads or GST control beads. Proteins bound to the beads were detected by immunoblotting with anti-V5, HA, or Myc tag antibodies as indicated. The input lane represents $5 \%$ of the total lysate. (B) S2 cells were transfected as indicated to express HA-tagged Ago $\Delta \mathrm{F}$ (Moberg et al. 2004), CycE-Renillla luciferase or Renilla luciferase, and GFP-Minus or GFP, as indicated. Lysates were immunoprecipitated with anti-HA, followed by release of bound complexes by incubation with HA peptide and immunoprecipitation with anti-GFP. Bound luciferase was measured. Error bars indicate standard deviation. $(C, D)$ Anti-dMyc labeling (red) of third instar wing discs containing clones of ago1 mutant or $\mathrm{mi}^{\mathrm{ALY}^{22}}$ mutant cells, as indicated. Mutant clones are labeled by the absence of GFP.

2003), as seen in flies carrying a leaky hsp70-Myc-CycE-I transgene (Fig. 5A,B). Consistent with the idea that reduced bristle size is due to excess CycE activity in the minus mutant, we observed that removing one copy of the $C y c E$ gene suppressed this phenotype (Fig. 5C,D). Fewer bristles were rescued in the combination carrying the weaker allele $C y c E^{5}$ than with the null allele $C y c E^{A r 95}$. The minus bristle phenotype was not affected by removing one copy of the $c d k 2$ gene, which is not the rate-limiting element of the CycE/Cdk2 complex.

Does excess CycE also impair the growth of minus mutant clones in diploid tissue? Clones of cells homozygous for the null allele $m i^{\Delta E Y 22}$ were typically eliminated 

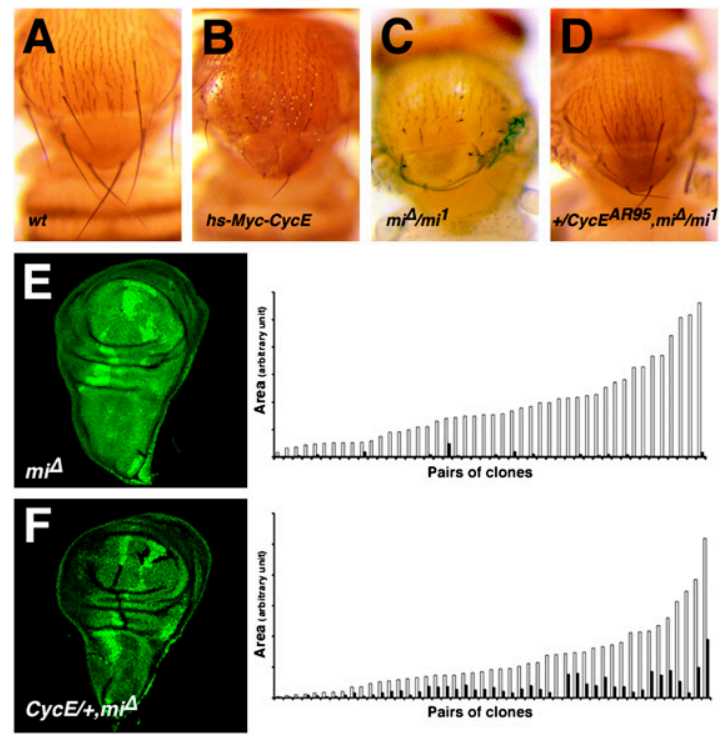

Figure 5. Some minus phenotypes are due to excess CycE. $(A-D)$ Adult dorsal thorax preparations of wild-type $(A)$, hsp $70-M y c-C y c E /+$ $(B), \mathrm{mi}^{\Delta E Y 22} / \mathrm{mi}^{1}(C)$, and $+/ C y c E^{A r 95}, \mathrm{mi}^{\Delta E Y 22} / \mathrm{mi}^{1}(D)$. (E, left panel): Third instar wing disc containing several twin spots (bright green) and a rare homozygous $m i^{\Delta E Y 22}$ mutant clone, marked by the absence of GFP. (Right panel) Histogram showing the area (arbitrary units) of 46 homozygous $\mathrm{mi}^{\mathrm{AEY} 22}$ mutant clones (shaded) and their twin spots. $(F$, left panel) Third instar wing disc heterozygote for $C y c E^{A r 95}$ containing several twin spots and homozygous $\mathrm{mi}^{\mathrm{AEY} 22}$ mutant clones, marked by the absence of GFP. (Right panel) Histogram showing the area of 47 homozygous $\mathrm{mi}^{\Delta E Y 22}$ mutant clones (shaded) and their twin spots. Although smaller on average than their twins, many more mutant clones were recovered than in the discs expressing two copies of the $C y c E$ gene.

from the imaginal discs by competition with fastergrowing Minus-expressing cells (Fig. 5E). As for the endoreplication defect, removing one copy of the $C y c E$ gene partially rescued survival and of growth these clones (Fig. 5F). Thus, excess CycE activity contributes to the defects in proliferation of diploid cells.

Our study provides evidence that efficient Cyclin E turnover is essential for normal cell proliferation and endoreplication during Drosophila development. Endoreplicative growth and more typical proliferative diploid cell cycles are impaired as a consequence of the elevated Cyclin E levels in minus mutants. Attempts to suppress the cell proliferation phenotype by reducing $\mathrm{CycE}$ activity were only partially successful, perhaps due to incomplete compensation for elevated CycE levels. It is also possible that Minus has other targets, in addition to CycE.

Minus was also identified in a screen for female sterility (Schüpbach and Wieschaus 1991). Minus' role in Cyclin E turnover suggests a possible link to the encore mutant. encore encodes a protein of unknown function that has been proposed to promote $\mathrm{CycE}$ degradation by localizing the SCF complex to a germline-specific cytoplasmic structure called the fusome (Ohlmeyer and Schüpbach 2003). In mammals, different Fbw7/Cdc4 isoforms can target different Myc functions in distinct subcellular compartments (Welcker and Clurman 2008). Interestingly, the Drosophila Fbw7/hCdc4 protein Archipelago exists in only one isoform (Moberg et al. 2001), limiting the possibility for isoform-specific subfunctions. Use of accessory proteins, such as Minus, may be another means to confer target specificity on the core Archipelago/SCF complex.

At present we cannot detect a Minus ortholog outside the insects. But, we note that Minus binds to human CCNE1 and influences its expression, much as it does with Drosophila CycE. Although this does not constitute evidence for the existence of a mammalian protein with a function analogous to Minus, it is compatible with this possibility. A vertebrate protein having the motifs required for Minus function but in a different number or arrangement might not be readily identified unless these short motifs were embedded in more extensive blocks of sequence similarity. In view of the importance of Cyclin E turnover in cancer (Hwang and Clurman 2005), a functional equivalent of Minus might be a good candidate for a tumor suppressor.

\section{Materials and methods}

\section{Fly strains}

The following mutant alleles were obtained from the Bloomington Stock Center: $y^{1}, w^{1118}, \mathrm{mi}^{1}, D f(2 L) b w-S 46, E Y 01258,1(2) \mathrm{k} 06908, K G 06805, \mathrm{da}$ GAL4, CycE $E^{5}, C y c E^{A r 95}, c d c 2 c^{2}, c d c 2 c^{3}, U A S-p 35, U A S-C y c E, U A S-G F P$, and ptc-GAL4. UAS-RNAi-mi lines were from the Vienna Drosophila RNAi center. 1(2)SH0818 was provided by S. Hou (Oh et al. 2003), FRT80B $a g O^{1}$ was provided by I. Hariharan; hs FLP; FRT42D, Ubi-GFP, M(2) $53^{1}$ was provided by D. Hipfner; and hh-GAL4, UAS-DIAP1 was provided by B.J. Thompson. $m i^{\triangle E Y 22}$ was generated by imprecise excision of EY01258. Mutants were balanced with $C y O, K r^{G A L 4}$, and UAS-GFP, and molecular characterization was done by PCR on GFP-negative larvae. Mutant clones were induced using the FLP/FRT system. Larvae were heat-shocked for $1 \mathrm{~h}$ at $38^{\circ} \mathrm{C}$ at $48 \pm 12 \mathrm{~h}$ in the following genotypes:

hs FLP/+ or Y; FRT42D, Ubi-GFP/FRT42D, mi ${ }^{\Delta E Y 22}$;

hs FLP/+ or Y; FRT42D, Ubi-GFP/FRT42D;

hs FLP/+ or Y; FRT42D, Ubi-GFP/ CycE ${ }^{A r 95}$, FRT42D, mi ${ }^{4 E Y 22}$; hs FLP/+ or Y; FRT42D, Ubi-GFP, M(2)53 ${ }^{1} /$ FRT42D, $\mathrm{mi}^{\Delta E Y 22}$;

hs FLP/+ or Y; FRT42D, Ubi-GFP, M(2)53 $1 /$ FRT42D;

hsFLP, UAS-CD8-GFP/+; FRT42D, tub-GAL80/FRT42D, $m i^{\Delta E Y 22} ;$ tub-GAL4/UAS-p35;

hs FLP/+ or Y; FRT42D, Ubi-GFP, M(2)53 ${ }^{1} / F R T 42 D, \mathrm{mi}^{\Delta E Y 22}$; hsp70-Myc-CycE/+; and

hs FLP/+ or Y; FRT80B, Ubi-GFP/FRT80B, ago ${ }^{1}$.

hs FLP/+ or Y; FRT42D, Ubi-GFP, M(2)53 $/ F R T 42 D, \mathrm{mi}^{\text {LEY22 }}$; hsp 70-Myc$C y c E /+$ larvae were heat-shocked for $1 \mathrm{~h}$ at $38^{\circ} \mathrm{C} 16 \mathrm{~h}$ before dissection to induce hsp 70-Myc-CycE expression. The hsp70-Myc-CycE transgene was derived from pIE1-4-myc-CycE (Moberg et al. 2001).

To produce salivary gland clones, 0 - to 6 -h embryos were heat-shocked for $1 \mathrm{~h}$ at $38^{\circ} \mathrm{C}$.

\section{Immunohistochemistry and FACS analysis}

Antibody staining of imaginal discs was performed as in Hipfner and Cohen (2003). The primary antibodies used were rabbit $\alpha$-CycE (C. Lehner), mouse $\alpha$-CycE (H. Richardson), monoclonal mouse $\alpha$-Myc P4C4-b10 (B. Edgar), and rabbit $\alpha$-phospho-Histone-H3 (Cell Signaling). FACS analysis was done according to Neufeld et al. (1998).

\section{Cell culture: dsRNA treatment, immunoprecipitation,} and luciferase assays

dsRNA was prepared using MegascriptT7 (Ambion) with templates dsRNA-1_f, 5' -taatacgactcactataggTCCTTTGGGCTGTGTCGC-3'; dsRNA-1_r, 5'-taatacgactcactataggTGGGTTGGCGTTCCTTGC-3'; and 
414-776: dsRNA-2_f, 5'-ctaatacgactcactatagggagCACGACAGCCACCA CGACAAC-3'; dsRNA-2_r, 5'-ctaatacgactcactatagggagTCCATCGTCTG CTCATCCACAAA-3'; nucleotides 17-633 of EGFP2, for ago as in Moberg et al. (2004). S2 cells $\left(2 \times 10^{6}\right)$ were treated with $12 \mu \mathrm{g}$ of dsRNA per well. Two days later, cells were transfected with $4 \mu \mathrm{g}$ of Cellfectin (Invitrogen) and $2 \mu \mathrm{g}$ of $p M T-m y c-C y E-I$ or $p M T-m y c-C y E-I I$ or $p M T-m y c-C C N E 1$. After $12 \mathrm{~h}$, the mix was replaced with medium containing $20 \mu \mathrm{g}$ of dsRNA. Cells were induced at $12 \mathrm{~h}$ with $0.7 \mathrm{mM} \mathrm{CuSO}_{4}$ for $2 \mathrm{~d}$. $p M T-m y c-$ CyE-I was derived from pIE1-4-myc-CycE (Moberg et al. 2001), and pMTmyc-CyE-II was made from EST LD22682. pMT-myc-CCNE1 was made using IRAUp969H0878D from Deutsches Ressourcenzentrum für Genomforschung GmbH.

For analysis of protein levels, cells were lysed in $50 \mathrm{mM}$ Tris (pH 7.5), $300 \mathrm{mM} \mathrm{NaCl}, 0.5 \%$ CHAPS, $25 \mathrm{mM} \mathrm{NaF}$, and protease inhibitor cocktail (Roche). Immunoblots were probed with rabbit $\alpha$-Myc (Santa Cruz Biotechnologies) or $\alpha$-Cyclin E (d300; Santa Cruz Biotechnologies) and $\alpha$-Kinesin (Cytoskeleton, Inc.) as a loading control.

For analysis of protein stability, cells were treated with dsRNA and transfected with $p M T-C y c E-R e n i l l a ~ l u c i f e r a s e$, but not induced with $\mathrm{CuSO}_{4}$. pMT-CyE-Renilla luciferase is a C-terminal fusion derived from pMT-myc-CyE-I. Cells were treated with cycloheximide $(10 \mu \mathrm{g} / \mathrm{mL})$ as indicated, and dual luciferase assays were according to the manufacturer's protocol (Promega).

For double immunoprecipitation, transfections were done with combinations of pMT-GFP-mi, pMT-GFP-mi, pMT-HA-Ago- $\Delta \mathrm{F}$ (Moberg et al. 2004), pMT-CyE-Renilla luciferase, or pMT-Renilla luciferase. pMT-GFP$\mathrm{mi}$ was generated by using $L D 45221$ and introducing EGFP2 (Clontech) at Minus residue 82. Cells were lysed in $20 \mathrm{mM}$ Tris (pH 7.5), $150 \mathrm{mM} \mathrm{NaCl}$, $0.2 \%$ NP- $40,10 \%$ glycerol, $1 \mathrm{mM}$ DTT, and protease and phophatase inhibitor cocktails (Roche). Lysates were immunoprecipitated with rat monoclonal 3F10 anti-HA (Roche) bound to protein G beads (Roche). Beads were washed five times in lysis buffer and incubated in lysis buffer with $0.5 \mathrm{mg} / \mathrm{mL}$ HA peptide (Roche). Eluates were immunoprecipitated with rabbit $\alpha$-GFP (Torrey Pines Biolabs) bound to protein A beads (Roche). Beads were washed four times in lysis buffer and Renilla luciferase activity was measured.

\section{GST pull-down assays}

GST-Minus (residues 82-725) was produced in Escherichia coli BL21 and purified on glutathione-sepharose beads. GST-Minus was incubated for $2 \mathrm{~h}$ at $4^{\circ} \mathrm{C}$ with lysates of $\mathrm{S} 2$ cells transfected with $p M T-m y c-C y c E-I$ or $p M T$ Myc-CCNE1 or pMT-V5-Ago (Ko et al. 2002) or pMT-HA-Skp1 (Zielke et al. 2006) or pAct-Myc-Cullin1 (Donaldson and Duronio, unpubl.). Beads were washed four times in $50 \mathrm{mM}$ Tris $(\mathrm{pH} 7.5), 100 \mathrm{mM} \mathrm{NaCl}$, and $0.5 \%$ CHAPS, and analyzed by immunoblotting with mouse 9E10 $\alpha$-Myc (Santa Cruz Biotechnologies), goat anti-V5 (Abcam), or rat 3F10 anti-HA (Roche).

\section{Acknowledgments}

We thank Ann-Mari Voie, Lidia Perez, Eva Loeser, and Andrew Riddell from the EMBL Flow Cytometry Core Facility for technical support; and members of the laboratory for support and helpful comments. S.S. was a recipient of a "La Ligue Nationale contre le Cancer" Post-Doctoral Fellowship and a Marie Curie Individual Fellowship. T.S. and V.H. were fellows of the Singapore Millennium Foundation. This work was supported by EMBL and by the Singapore Millennium Foundation via core funding to T.L.L.

\section{References}

Du W, Vidal M, Xie JE, Dyson N. 1996. RBF, a novel RB-related gene that regulates E2F activity and interacts with cyclin E in Drosophila. Genes \& Dev 10: 1206-1218.

FlyBase Consortium. 2003. The FlyBase database of the Drosophila genome projects and community literature. Nucleic Acids Res 31: 172-175.

Follette PJ, Duronio RJ, O'Farrell PH. 1998. Fluctuations in cyclin E levels are required for multiple rounds of endocycle $S$ phase in Drosophila. Curr Biol 8: 235-238.
Giot L, Bader JS, Brouwer C, Chaudhuri A, Kuang B, Li Y, Hao YL, Ooi CE, Godwin B, Vitols E, et al. 2003. A protein interaction map of Drosophila melanogaster. Science 302: 1727-1736.

Hay BA, Wolff T, Rubin GM. 1994. Expression of baculovirus P35 prevents cell death in Drosophila. Development 120: 2121-2129.

Hipfner DR, Cohen SM. 2003. The Drosophila sterile-20 kinase slik controls cell proliferation and apoptosis during imaginal disc development. PLOS Biol 1: E35. doi: 10.1371/journal.pbio.0000035.

Hwang HC, Clurman BE. 2005. Cyclin E in normal and neoplastic cell cycles. Oncogene 24: 2776-2786.

Johnston LA, Prober DA, Edgar BA, Eisenman RN, Gallant P. 1999. Drosophila myc regulates cellular growth during development. Cell 98: 779-790.

Knoblich JA, Sauer K, Jones L, Richardson H, Saint R, Lehner CF. 1994. Cyclin E controls $\mathrm{S}$ phase progression and its down-regulation during Drosophila embryogenesis is required for the arrest of cell proliferation. Cell 77: 107-120.

Ko HW, Jiang J, Edery I. 2002. Role for Slimb in the degradation of Drosophila Period protein phosphorylated by Doubletime. Nature 420: 673-678.

Lane ME, Elend M, Heidmann D, Herr A, Marzodko S, Herzig A, Lehner CF. 2000. A screen for modifiers of cyclin E function in Drosophila melanogaster identifies Cdk2 mutations, revealing the insignificance of putative phosphorylation sites in Cdk2. Genetics 155: 233-244.

Lilly MA, Duronio RJ. 2005. New insights into cell cycle control from the Drosophila endocycle. Oncogene 24: 2765-2775.

Lindsley DL, Zimm GG. 1992. The genome of Drosophila melanogaster. Academic Press, San Diego.

Moberg KH, Bell DW, Wahrer DC, Haber DA, Hariharan IK. 2001. Archipelago regulates Cyclin E levels in Drosophila and is mutated in human cancer cell lines. Nature 413: 311-316.

Moberg KH, Mukherjee A, Veraksa A, Artavanis-Tsakonas S, Hariharan IK. 2004. The Drosophila F box protein archipelago regulates dMyc protein levels in vivo. Curr Biol 14: 965-974.

Morata G, Ripoll P. 1975. Minutes: Mutants of Drosophila autonomously affecting cell division rate. Dev Biol 42: 211-221.

Mortimer NT, Moberg KH. 2007. The Drosophila F-box protein Archipelago controls levels of the Trachealess transcription factor in the embryonic tracheal system. Dev Biol 312: 560-571.

Murray AW. 2004. Recycling the cell cycle: Cyclins revisited. Cell 116: 221-234.

Neufeld TP, de la Cruz AF, Johnston LA, Edgar BA. 1998. Coordination of growth and cell division in the Drosophila wing. Cell 93: 1183-1193.

Oh SW, Kingsley T, Shin HH, Zheng Z, Chen HW, Chen X, Wang H, Ruan P, Moody M, Hou SX. 2003. A P-element insertion screen identified mutations in 455 novel essential genes in Drosophila. Genetics 163: 195-201.

Ohlmeyer JT, Schüpbach T. 2003. Encore facilitates SCF-Ubiquitinproteasome-dependent proteolysis during Drosophila oogenesis. Development 130: 6339-6349.

Richardson HE, O'Keefe LV, Reed SI, Saint R. 1993. A Drosophila G1specific cyclin $\mathrm{E}$ homolog exhibits different modes of expression during embryogenesis. Development 119: 673-690.

Schüpbach T, Wieschaus E. 1991. Female sterile mutations on the second chromosome of Drosophila melanogaster. II. Mutations blocking oogenesis or altering egg morphology. Genetics 129: 1119-1136.

Su TT, Sprenger F, DiGregorio PJ, Campbell SD, O'Farrell PH. 1998. Exit from mitosis in Drosophila syncytial embryos requires proteolysis and cyclin degradation, and is associated with localized dephosphorylation. Genes \& Dev 12: 1495-1503.

Weiss A, Herzig A, Jacobs H, Lehner CF. 1998. Continuous Cyclin E expression inhibits progression through endoreduplication cycles in Drosophila. Curr Biol 8: 239-242.

Welcker M, Clurman BE. 2008. FBW7 ubiquitin ligase: A tumour suppressor at the crossroads of cell division, growth and differentiation. Nat Rev Cancer 8: 83-93.

Weng L, Zhu C, Xu J, Du W. 2003. Critical role of active repression by E2F and $\mathrm{Rb}$ proteins in endoreplication during Drosophila development. EMBO J 22: 3865-3875.

Zielke N, Querings S, Grosskortenhaus R, Reis T, Sprenger F. 2006. Molecular dissection of the APC/C inhibitor Rcal shows a novel F-box-dependent function. EMBO Rep 7: 1266-1272. 


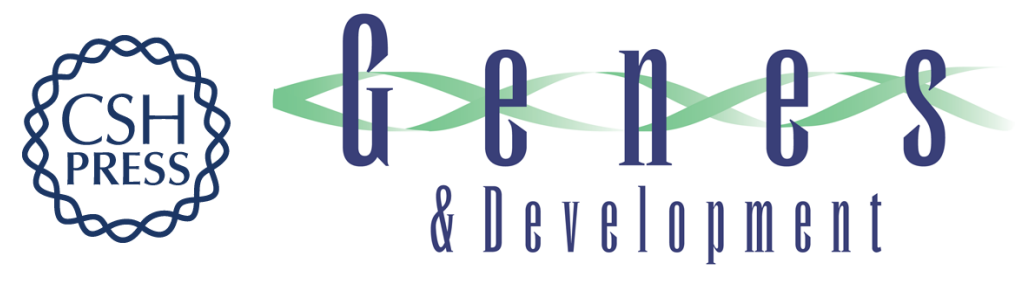

\section{Drosophila Minus is required for cell proliferation and influences Cyclin E turnover}

Sébastien Szuplewski, Thomas Sandmann, Ville Hietakangas, et al.

Genes Dev. 2009, 23:

Access the most recent version at doi:10.1101/gad.1822409

Supplemental http://genesdev.cshlp.org/content/suppl/2009/08/14/23.17.1998.DC1
Material

References This article cites 27 articles, 10 of which can be accessed free at: http://genesdev.cshlp.org/content/23/17/1998.full.html\#ref-list-1

License Freely available online through the Genes \& Development Open Access option.

Email Alerting
Service $\begin{aligned} & \text { Receive free email alerts when new articles cite this article - sign up in the box at the top } \\ & \text { right corner of the article or click here. }\end{aligned}$ 